General Mathematics Vol. 27, No. 1 (2019), 71-84

DOI:10.2478/gm-2019-0007

S sciendo

\title{
A study of fractional integro-differential equations via Hilfer-Hadamard fractional derivative ${ }^{1}$
}

\author{
D. Vivek, K. Kanagarajan, E. M. Elsayed
}

\begin{abstract}
In this paper, we investigate the existence of solution of integro-differential equations (IDEs) with Hilfer-Hadamard fractional derivative. The main results are obtained by using Schaefer's fixed point theorem. Some Ulam stability results are presented.
\end{abstract}

2010 Mathematics Subject Classification: 26A33, 60H10, 34K30, 34K40.

Key words and phrases: Fractional integro-differential equations; Hadamard fractional derivative; Hilfer fractional derivative; Solution; Ulam-Hyers stablity.

\section{Introduction}

It is familiar that the fractional derivatives are helpful tools for the description of memory and hereditary properties of various material sand processes, which integer order derivatives can't characterize. Several problems in various fields can be described by fractional calculus such as material sciences, mechanics, wave propagation, signal processing, and system identification and so on. The fractional differential equations have gained considerable importance during the past three decades. Hence, the theory of fractional differential equations has emerged as an active branch of applied mathematics. It has been used to construct many mathematical models in various fields, such as physics, chemistry, viscoelasticity, electrochemistry, control, porous media, electromagnetic and polymer rheology, etc. The recent works on the theory and application of fractional differential equations, we refer to the books $[27,30,31,32]$. Hilfer proposed a generalized Riemann-Liouville fractional derivative for short, Hilfer fractional derivative, which includes Riemann-Liouville fractional derivative and Caputo fractional derivative (see [1, 13, 14, 15, 28, 29, 33]).

\footnotetext{
${ }^{1}$ Received 1 October, 2017

Accepted for publication (in revised form) 8 May, 2019
} 
In recent years, attention has been paid to establish sufficient conditionsfor the existenceresults to differentialsystems involving Hilferfractional derivatives. Subsequently, many authors studied the fractional differential equations involving Hilfer fractional derivatives. Recently, the study of Hilfer-Hadamard fractional derivativefor (short H-H derivative) of differential equations is also of great importance. There has been a significant development in $\mathrm{H}-\mathrm{H}$ derivative of differential equations in recent years $[11,23,2,3]$. For detail study on Hadamard fractional derivative,we refer to $[4,11,12]$.

In addition, V. Gupta and J. Dabas discussed the existence of solution for fractional impulsive IDEs with integral boundary conditions in [16]. The authors also studied the existence results of solutions for impulsive fractional differential equations in [17]. In [10], the existence of solutions of fractional IDEs by using the resolvent operators and fixed point theorem are proved. Existence results for boundary value problems of arbitrary order IDEs in Banach spaces was studied by K. Karthikeyan and Bashir Ahmad [21]. For some investigations on fractional IDEs, one can refer to $[5,8,9,22]$.

In the present work, we consider an initial value problem of IDEs with $\mathrm{H}-\mathrm{H}$ derivative given by

$$
\begin{aligned}
& { }_{H} D_{1^{+}}^{\alpha, \beta} x(t)=f\left(t, x(t), \int_{1}^{t} h(t, s, x(s)) \frac{d s}{s}\right), \quad t \in J:=[1, T], \\
& \left.{ }_{H} I_{1^{+}}^{1-\gamma} x(t)\right|_{t=1}=x_{0}, \quad \gamma=\alpha+\beta-\alpha \beta
\end{aligned}
$$

where ${ }_{H} D_{1^{+}}^{\alpha, \beta}$ is the $\mathrm{H}-\mathrm{H}$ fractional derivative, $0<\alpha<1,0 \leq \beta \leq 1$. Let $X$ be a Banach space, $f: J \times X \times X \rightarrow X, h: \Delta \times X \rightarrow X$ are given continuous functions and $I_{1+}^{1-\gamma}$ is generalized Hadamard fractional integral of order $1-\gamma$. For brevity of notation, we shall take ${ }_{H} I_{1^{+}}^{1-\gamma}$ as $I_{1^{+}}^{1-\gamma}$. Here $\Delta=\{(t, s): 0 \leq s \leq t \leq T\}$. For brevity, let us take

$$
H x(t)=\int_{1}^{t} h(t, s, x(s)) \frac{d s}{s} .
$$

It is seen that equation (1) is equivalent to the integral equation

$$
x(t)=\frac{x_{0}}{\Gamma(\gamma)}(\log t)^{\gamma-1}+\frac{1}{\Gamma(\alpha)} \int_{1}^{t}\left(\log \frac{t}{s}\right)^{\alpha-1} f(s, x(s), H x(s)) \frac{d s}{s} .
$$

This paper is organized as follows. In Section 2, we introduce some preliminary results needed in the developing of the paper. In Section 3, we present an existence result for initial value problem for fractional IDEs with $\mathrm{H}-\mathrm{H}$ derivative and stability result is discussed in Section 4.

\section{Preliminaries}

In this section, we introduce some basic definitions, propositions and lemmas, which will be used throughout the work. 
Let $C[J, X]$ denotes the Banach space of continuous function on $[1, T]$ with the norm

$$
\|x\|_{C}:=\sup \{x(t): t \in J\} .
$$

We denote $L^{1}\left\{R_{+}\right\}$, the space of Lebesgue integrable functions on J. In order to define the solution of (1), we shall consider $C_{\gamma, \log }[J, X]$ is the weighted space of the continuous function

$$
C_{\gamma, \log }[J, X]:=\left\{f(t): J \rightarrow X \mid(\log t)^{\gamma} f(t) \in C[J, X]\right\},
$$

with norm

$$
\|f\|_{C_{\gamma, \log }}=\left\|(\log t)^{\gamma} f(t)\right\|_{C},
$$

and

$$
\|f\|_{C_{\gamma}^{n}}=\sum_{k=0}^{n-1}\left\|f^{k}\right\|_{C}+\left\|f^{n}\right\|_{C_{\gamma, \log }} .
$$

Moreover, $C_{\gamma}^{0}[J, X]:=C_{\gamma, \log }[J, X]$.

In order to solve our problem, the following spaces are presented.

$$
C_{1-\gamma, \log }^{\alpha, \beta}[J, X]=\left\{f \in C_{1-\gamma, \log }[J, X],_{H} D_{1^{+}}^{\alpha, \beta} f \in C_{1-\gamma, \log }[J, X]\right\}
$$

and

$$
C_{1-\gamma, \log }^{\gamma}[J, X]=\left\{f \in C_{1-\gamma, \log }[J, X],_{H} D_{1^{+}}^{\gamma} f \in C_{1-\gamma, \log }[J, X]\right\} .
$$

It is obvious that

$$
C_{1-\gamma, \log }^{\gamma}[J, X] \subset C_{1-\gamma, \log }^{\alpha, \beta}[J, X] .
$$

For basic facts about fractional derivative and fractional calculus one can refer the books [27, 30, 32].

Definition 1. The Hadamard derivative of fractional order $\alpha$ for a function $f$ : $[1, \infty) \rightarrow X$ is defined as

$D^{\alpha} f(t)=\frac{1}{\Gamma(n-\alpha)}\left(t \frac{d}{d t}\right)^{n} \int_{1}^{t}\left(\log \frac{t}{s}\right)^{n-\alpha-1} f(s) \frac{d s}{s}, \quad n-1<\alpha<n, n=\lceil\alpha\rceil+1$,

where $\lceil\alpha\rceil$ denotes the integer part of real number $\alpha$ and $\log (\cdot)=\log _{e}(\cdot)$.

Definition 2. The Hadamard fractional integral of order $\alpha$ for a function $f$ is defined as

$$
I^{\alpha} f(t)=\frac{1}{\Gamma(\alpha)} \int_{1}^{t}\left(\log \frac{t}{s}\right)^{\alpha-1} f(s) \frac{d s}{s}, \quad \alpha>0,
$$

provided the integral exists. 
Definition 3. (H-H fractional derivative) The left-sided Hilfer-Hadamard fractional derivative of order $0<\alpha<1$ and $0 \leq \beta \leq 1$ of function $h(x)$ is defined by

$$
{ }_{H} D_{1^{+}}^{\alpha, \beta} h(t)=\left(I_{1^{+}}^{\beta(1-\alpha)} D\left(I_{1^{+}}^{(1-\beta)(1-\alpha)} h\right)\right)(t), \quad D=\frac{d}{d t} .
$$

The generalized Riemann-Liouville Hadamard fractional derivative is used as an interpolator between the Riemann-Liouville and Caputo Hadamard fractional derivative.

\section{Existence results}

This section deals with the existence and uniqueness of solutions for the problem (1).

Before stating and proving the main results, we introduce the following assumptions.

(A1) The function $f: J \times X \times X \rightarrow X$ is continuous.

(A2) The function $f: J \times X \times X \rightarrow X$ is completely continuous and there exists a function $\mu \in L^{1}\left\{R_{+}\right\}$such that

$$
|f(t, u, v)| \leq \mu(t), \quad \forall t \in J, \quad u, v \in X .
$$

(A3) The function $f: J \times X \times X \rightarrow X$ is continuous and there exists a constant $L_{1}>0$ such that

$$
\left|f\left(t, x_{1}, y_{1}\right)-f\left(t, x_{2}, y_{2}\right)\right| \leq L_{1}\left[\left|x_{1}-x_{2}\right|+\left|y_{1}-y_{2}\right|\right], \quad \forall t \in J, x_{1}, x_{2}, y_{1}, y_{2} \in X .
$$

(A4) The function $h: \Delta \times X \rightarrow X$ is continuous and there exists a constant $H_{1}>0$ such that

$$
\left|h\left(t, s, x_{1}\right)-h\left(t, s, x_{2}\right)\right| \leq H_{1}\left|x_{1}-x_{2}\right|, \quad \forall t \in J, x_{1}, x_{2} \in X .
$$

Theorem 1. Assume that (A1)-(A2) hold. Then, the problem (1) has at least one solution on $J$.

Proof. The proof will be given in the following sequence.

Let $C=C_{1-\gamma, \log }[J, X]$. Consider the operator $N: C \rightarrow C$ defined by

(3) $\quad(N x)(t)=\frac{x_{0}}{\Gamma(\gamma)}(\log t)^{\gamma-1}+\frac{1}{\Gamma(\alpha)} \int_{1}^{t}\left(\log \frac{t}{s}\right)^{\alpha-1} f(s, x(s), H x(s)) \frac{d s}{s}$.

We shall show that the operator $N$ is continuous and completely continuous.

Step 1. $N$ is continuous. 
Let $x_{n}$ be a sequence such that $x_{n} \rightarrow x$ in $C$. Then for each $t \in J$

$$
\begin{aligned}
& \left|(\log t)^{1-\gamma}\left(\left(N x_{n}\right)(t)-(N x)(t)\right)\right| \\
& \leq \frac{(\log t)^{1-\gamma}}{\Gamma(\alpha)} \int_{1}^{t}\left(\log \frac{t}{s}\right)^{\alpha-1}\left|f\left(s, x_{n}(s), H x_{n}(s)\right)-f(s, x(s), H x(s))\right| \frac{d s}{s} \\
& \leq \frac{(\log t)^{1-\gamma}}{\Gamma(\alpha)} \int_{1}^{t}\left(\log \frac{t}{s}\right)^{\alpha-1} \sup _{s \in J}\left|f\left(s, x_{n}(s), H x_{n}(s)\right)-f(s, x(s), H x(s))\right| \frac{d s}{s} \\
& \leq \frac{(\log T)^{1-\gamma+\alpha}}{\Gamma(\alpha+1)}\left\|f\left(\cdot, x_{n}(\cdot), H x_{n}(\cdot)\right)-f(\cdot, x(\cdot), H x(\cdot))\right\|_{C} .
\end{aligned}
$$

Since $f$ is a continuous function, we have

$$
\begin{aligned}
\left\|N x_{n}-N x\right\|_{C} & \leq \frac{(\log T)^{1-\gamma+\alpha}}{\Gamma(\alpha+1)}\left\|f\left(\cdot, x_{n}(\cdot), H x_{n}(\cdot)\right)-f(\cdot, x(\cdot), H x(\cdot))\right\|_{C} \\
& \rightarrow 0 \quad \text { as } \quad n \rightarrow \infty .
\end{aligned}
$$

Step 2. $N$ maps bounded sets into bounded sets in $C$.

Indeed, it is enough to show that $q>0$, there exists a positive constant $l$ such that $x \in B_{q}=\{x \in C:\|x\| \leq q\}$, we have $\|N x\|_{C} \leq l$.

$$
\begin{aligned}
& \left|(\log t)^{1-\gamma}(N x)(t)\right| \\
& \leq \frac{\left|x_{0}\right|}{\Gamma(\gamma)}+\frac{(\log t)^{1-\gamma}}{\Gamma(\alpha)} \int_{1}^{t}\left(\log \frac{t}{s}\right)^{\alpha-1}|f(s, x(s), H x(s))| \frac{d s}{s} \\
& \leq \frac{\left|x_{0}\right|}{\Gamma(\gamma)}+\frac{(\log t)^{1-\gamma}}{\Gamma(\alpha)} \int_{1}^{t}\left(\log \frac{t}{s}\right)^{\alpha-1}|\mu(s)| \frac{d s}{s} \\
& \leq \frac{\left|x_{0}\right|}{\Gamma(\gamma)}+\frac{(\log T)^{1-\gamma+\alpha}}{\Gamma(\alpha+1)}\|\mu\|_{C} .
\end{aligned}
$$

Step 3. $N$ maps bounded sets into equicontinuous set of $C$.

Let $t_{1}, t_{2} \in J, t_{1}<t_{2}, B_{q}$ be a bounded set of $C$ as in Step 2, and $x \in B_{q}$. Then

$$
\begin{aligned}
& \left|\left(\log t_{2}\right)^{1-\gamma}(N x)\left(t_{2}\right)-\left(\log t_{1}\right)^{1-\gamma}(N x)\left(t_{1}\right)\right| \\
& \leq \mid \frac{\left(\log t_{2}\right)^{1-\gamma}}{\Gamma(\alpha)} \int_{1}^{t_{2}}\left(\log \frac{t_{2}}{s}\right)^{\alpha-1} f(s, x(s), H x(s)) \frac{d s}{s} \\
& -\frac{\left(\log t_{1}\right)^{1-\gamma}}{\Gamma(\alpha)} \int_{1}^{t_{1}}\left(\log \frac{t_{1}}{s}\right)^{\alpha-1} f(s, x(s), H x(s)) \frac{d s}{s} \mid \\
& \leq \frac{\|\mu\|_{C}}{\Gamma(\alpha)} \int_{1}^{t_{1}}\left|\left(\log t_{2}\right)^{1-\gamma}\left(\log \frac{t_{2}}{s}\right)^{\alpha-1}-\left(\log t_{1}\right)^{1-\gamma}\left(\log \frac{t_{1}}{s}\right)^{\alpha-1}\right| \frac{d s}{s} \\
& +\frac{\left(\log t_{2}\right)^{1-\gamma}}{\Gamma(\alpha+1)}\left(\log \frac{t_{2}}{t_{1}}\right)^{\alpha}\|\mu\|_{C} .
\end{aligned}
$$


As $t_{1} \rightarrow t_{1}$, the right hand side of the above inequality tends to zero. As a consequence of Step 1 to 3, together with Arzela-Ascoli theorem, we can conclude that $N: C \rightarrow C$ is continuous and completely continuous.

Step 4. A priori bounds.

Now it remains to show that the set

$$
\omega=\{x \in C: X=\delta(N x), \quad \delta \in(0,1)\}
$$

is bounded set.

Let $x \in \omega, x=\delta(N x)$ for some $0<\delta<1$. Thus for each $t \in J$, we have

$$
x(t)=\delta\left[\frac{x_{0}}{\Gamma(\gamma)}(\log t)^{\gamma-1}+\frac{1}{\Gamma(\alpha)} \int_{1}^{t}\left(\log \frac{t}{s}\right)^{\alpha-1} f(s, x(s), H x(s)) \frac{d s}{s}\right] .
$$

This implies by (A2) that for each $t \in J$, we have

$$
\begin{aligned}
\left|x(t)(\log t)^{1-\gamma}\right| & \leq\left|(\log t)^{1-\gamma}(N x)(t)\right| \\
& \leq \frac{\left|x_{0}\right|}{\Gamma(\gamma)}+\frac{(\log t)^{1-\gamma}}{\Gamma(\alpha)} \int_{1}^{t}\left(\log \frac{t}{s}\right)^{\alpha-1}|f(s, x(s), H x(s))| \frac{d s}{s} \\
& \leq \frac{\left|x_{0}\right|}{\Gamma(\gamma)}+\frac{(\log t)^{1-\gamma}}{\Gamma(\alpha)} \int_{1}^{t}\left(\log \frac{t}{s}\right)^{\alpha-1}|\mu(s)| \frac{d s}{s} \\
& \leq \frac{\left|x_{0}\right|}{\Gamma(\gamma)}+\frac{(\log T)^{1-\gamma+\alpha}}{\Gamma(\alpha+1)}\|\mu\|_{C} \\
& \leq R
\end{aligned}
$$

that $\|\mu(t)\|_{C} \leq R$.

This shows that the set $\omega$ is bounded. As a consequence of Schaefer's fixed point theorem, we deduce that $N$ has a fixed point which is a solution of problem (1).

Finally, we give the following uniqueness result.

Theorem 2. Assume that (A3) and (A4) hold. If

$$
\left(\frac{(\log T)^{1-\gamma+\alpha}}{\Gamma(\alpha+1)} L_{1}\left(1+H_{1}\right)\right)<1
$$

then the problem (1) has a unique solution.

Consider the operator $N: C \rightarrow C$ defined by

(5) $(N x)(t)=\frac{x_{0}}{\Gamma(\gamma)}(\log t)^{\gamma-1}+\frac{1}{\Gamma(\alpha)} \int_{1}^{t}\left(\log \frac{t}{s}\right)^{\alpha-1} f(s, x(s), H x(s)) \frac{d s}{s}$. 
It is clear that the fixed points of $N$ are solutions of problem (1).

Let $x_{1}, x_{2} \in C$ and $t \in J$, then we have

$$
\begin{aligned}
& \left|(\log t)^{1-\gamma}\left(\left(N x_{1}\right)(t)-\left(N x_{2}\right)(t)\right)\right| \\
& \leq \frac{(\log t)^{1-\gamma}}{\Gamma(\alpha)} \int_{1}^{t}\left(\log \frac{t}{s}\right)^{\alpha-1}\left|f\left(s, x_{1}(s), H x_{1}(s)\right)-f\left(s, x_{2}(s), H x_{2}(s)\right)\right| \frac{d s}{s} \\
& \leq \frac{(\log t)^{1-\gamma}}{\Gamma(\alpha)} \int_{1}^{t}\left(\log \frac{t}{s}\right)^{\alpha-1} L_{1}\left(\left|x_{1}(s)-x_{2}(s)\right|+\left|H x_{1}-H x_{2}\right|\right) \frac{d s}{s} \\
& \leq\left(\frac{(\log T)^{1-\gamma+\alpha}}{\Gamma(\alpha+1)} L_{1}\left(1+H_{1}\right)\right)\left\|x_{1}-x_{2}\right\|_{C} .
\end{aligned}
$$

Then

$$
\left\|N x_{1}-N x_{2}\right\|_{C} \leq\left(\frac{(\log T)^{1-\gamma+\alpha}}{\Gamma(\alpha+1)} L_{1}\left(1+H_{1}\right)\right)\left\|x_{1}-x_{2}\right\|_{C} .
$$

From (4), it follows that $N$ has a unique fixed point which is solution of problem (1).

\section{Ulam stability results}

The stability of functional equations was originally raised by Ulam in 1940 in a talk given at the University of Wisconsin. The first answer to Ulam's question was given by Hyers in 1941 in the case of Banach spaces. Thereafter, this type of stability is called Ulam-Hyers stability;one can refer to [7, 18, 19, 24]. In 1978, Rassias [26] provided a remarkable generalization of the Ulam-Hyers stability of mappings by considering variables. The concept of stability for a functional equation arises when we replace the functional equation by an inequality which acts as a perturbation of the equation. Ulam stability for fractional differential equations with Caputo and Riemann-Liouville derivative were proposed by some researchers. More details, from a historical point of view, and recent developments of such stabilities are reported in $[18,26,34,35]$.

In this section, we consider the Ulam stability of IDEs with $\mathrm{H}-\mathrm{H}$ fractional derivative (1).

Definition 4. [33] The equation (1) is Ulam-Hyers stable if there exists a real number $C_{f}>0$ such that for each $\epsilon>0$ and for each solution $z \in C_{1-\gamma, \log }^{\gamma}[J, X]$ of the inequality

$$
\left|{ }_{H} D_{1^{+}}^{\alpha, \beta} z(t)-f(t, z(t), H z(t))\right| \leq \epsilon, \quad t \in J,
$$

there exists a solution $x \in C_{1-\gamma, \log }^{\gamma}[J, X]$ of equation (1) with

$$
|z(t)-x(t)| \leq C_{f} \epsilon, \quad t \in J .
$$


Definition 5. [33] The equation (1) is generalized Ulam-Hyers stable if there exists $\psi_{f} \in C([0, \infty),[0, \infty)), \psi_{f}(0)=0$ such that for each solution $z \in C_{1-\gamma, \log }^{\gamma}[J, X]$ of the inequality

$$
\left|{ }_{H} D_{1^{+}}^{\alpha, \beta} z(t)-f(t, z(t), H z(t))\right| \leq \epsilon, \quad t \in J,
$$

there exists a solution $x \in C_{1-\gamma, \log }^{\gamma}[J, X]$ of equation (1) with

$$
|z(t)-x(t)| \leq \psi_{f} \epsilon, \quad t \in J .
$$

Definition 6. [33] The equation (1) is Ulam-Hyers-Rassias stable with respect to $\varphi \in C_{1-\gamma, \log }[J, X]$ if there exists a real number $C_{f}>0$ such that for each $\epsilon>0$ and for each solution $z \in C_{1-\gamma, \log }^{\gamma}[J, X]$ of the inequality

$$
\left|{ }_{H} D_{1^{+}}^{\alpha, \beta} z(t)-f(t, z(t), H z(t))\right| \leq \epsilon \varphi(t), \quad t \in J,
$$

there exists a solution $x \in C_{1-\gamma, \log }^{\gamma}[J, X]$ of equation (1) with

$$
|z(t)-x(t)| \leq C_{f} \epsilon \varphi(t), \quad t \in J .
$$

Definition 7. [33] The equation (1) is generalized Ulam-Hyers-Rassias stable with respect to $\varphi \in C_{1-\gamma, \log }[J, X]$ if there exists a real number $C_{f, \varphi}>0$ such that for each solution $z \in C_{1-\gamma, \log }^{\gamma}[J, X]$ of the inequality

$$
\left|{ }_{H} D_{1^{+}}^{\alpha, \beta} z(t)-f(t, z(t), H z(t))\right| \leq \varphi(t), \quad t \in J,
$$

there exists a solution $x \in C_{1-\gamma, \log }^{\gamma}[J, X]$ of equation (1) with

$$
|z(t)-x(t)| \leq C_{f, \varphi} \varphi(t), \quad t \in J
$$

Remark 1. A function $z \in C_{1-\gamma, \log }^{\gamma}[J, X]$ is a solution of the inequality (6) if and only if there exists a function $g \in C_{1-\gamma, \log }^{\gamma}[J, X]$ (which depend on solution $x$ ) such that
1. $|g(t)| \leq \epsilon, t \in J$
2. ${ }_{H} D_{1^{+}}^{\alpha, \beta} z(t)=f(t, z(t), H z(t))+g(t), t \in J$.

Remark 2. It is clear that:

1. Definition $4 \Rightarrow$ Definition 5.

2. Definition $6 \Rightarrow$ Definition $\%$.

Lemma 1. If a function $z \in C_{1-\gamma}^{\gamma}[J, X]$ is a solution of the inequality (6), then $z$ is a solution of the following integral inequality

$$
\left|z(t)-x_{0}(t)-\frac{1}{\Gamma(\alpha)} \int_{1}^{t}\left(\log \frac{t}{s}\right)^{\alpha-1} f(s, z(s), H z(s)) \frac{d s}{s}\right| \leq \frac{\epsilon(\log T)^{\alpha}}{\Gamma(\alpha+1)}
$$

with $x_{0}(t)=\frac{x_{0}}{\Gamma(\gamma)}(\log t)^{1-\gamma}$. 
Proof. The proof directly follows from Remark 1 and equation (2).

We have similar remarks for the solutions of the inequalities (8) and (9).

Now, we give the main results, generalized Ulam-Hyers-Rassias stable results, in this section.

Lemma 2. [36] Suppose $1>\alpha>0, \bar{a}>0$ and $\bar{b}>0$ and suppose $u(t)$ is nonnegative and locally integral on $[1,+\infty)$ with

$$
u(t) \leq \bar{a}+\bar{b} \int_{1}^{t}\left(\log \frac{t}{s}\right)^{\alpha-1} u(s) \frac{d s}{s}, \quad t \in[1,+\infty) .
$$

Then

$$
u(t) \leq \bar{a}+\int_{1}^{t}\left[\sum_{n=1}^{\infty} \frac{(\bar{b} \Gamma(\alpha))^{n}}{\Gamma(n \alpha)}\left(\log \frac{t}{s}\right)^{n \alpha-1} \bar{a}\right] \frac{d s}{s}, \quad t \in[1,+\infty) .
$$

Remark 3. Under the assumptions of Lemma 2, let $u(t)$ be a nondecreasing function on $[1, \infty)$. Then we have

$$
u(t) \leq \bar{a} E_{\alpha, 1}\left(\bar{b} \Gamma(\alpha)(\log t)^{\alpha}\right),
$$

where $E_{\alpha, 1}$ is the Mittag-leffler function defined by

$$
E_{\alpha, 1}(z)=\sum_{k=0}^{\infty} \frac{z^{k}}{\Gamma(k \alpha+1)}, \quad z \in \mathbb{C} .
$$

Theorem 3. Assume that (A1),(A3),(A4) and (4) hold. Then the problem (1) is Ulam-Hyers stable.

Proof. Let $\epsilon>0$ and let $z \in C_{1-\gamma, \log }^{\gamma}[J, X]$ be a function which satisfies the inequality (6) and let $x \in C_{1-\gamma, \log }^{\gamma}[J, X]$ be the unique solution of the following problem

$$
\begin{aligned}
& { }_{H} D_{1^{+}}^{\alpha, \beta} x(t)=f(t, x(t), H x(t)), t \in J:=[1, T], \\
& \left.I_{1^{+}}^{1-\gamma} x(t)\right|_{t=1}=\left.I_{1^{+}}^{1-\gamma} z(t)\right|_{t=1}=x_{0}, \quad \gamma=\alpha+\beta-\alpha \beta,
\end{aligned}
$$

where $0<\alpha<1$ and $0 \leq \beta \leq 1$.

We obtain the integral equation as follows:

$$
x(t)=x_{0}(t)+\left[I_{1^{+}}^{\alpha} f(s, x(s), H x(s))\right](t) .
$$

with $\frac{x_{0}}{\Gamma(\gamma)}(\log t)^{\gamma-1}$.

By integration of the inequality (6) and from Lemma 1, we obtain

$$
\left|z(t)-x_{0}(t)-\frac{1}{\Gamma(\alpha)} \int_{1}^{t}\left(\log \frac{t}{s}\right)^{\alpha-1} f(s, z(s), H z(s)) \frac{d s}{s}\right| \leq \frac{\epsilon(\log T)^{\alpha}}{\Gamma(\alpha+1)} .
$$


We have

$$
\begin{aligned}
& |z(t)-x(t)| \\
& \leq\left|z(t)-x_{0}(t)-\frac{1}{\Gamma(\alpha)} \int_{1}^{t}\left(\log \frac{t}{s}\right)^{\alpha-1} f(s, z(s), H z(s)) \frac{d s}{s}\right| \\
& \quad+\left|\frac{1}{\Gamma(\alpha)} \int_{1}^{t}\left(\log \frac{t}{s}\right)^{\alpha-1}[f(s, z(s), H z(s))-f(s, x(s), H x(s))] \frac{d s}{s}\right| \\
& \leq \frac{\epsilon(\log T)^{\alpha}}{\Gamma(\alpha+1)}+\frac{L_{1}\left(1+H_{1}\right)}{\Gamma(\alpha)} \int_{1}^{t}\left(\log \frac{t}{s}\right)^{\alpha-1}|z(s)-x(s)| \frac{d s}{s},
\end{aligned}
$$

and to apply Lemma 2 and Remark 3, we obtain

$$
\begin{aligned}
|z(t)-x(t)| & \leq \frac{(\log T)^{\alpha} E_{\alpha, 1}\left(L_{1}\left(1+H_{1}\right)(\log T)^{\alpha}\right)}{\Gamma(\alpha+1)} \cdot \epsilon \\
& :=C_{f} \epsilon .
\end{aligned}
$$

Thus, the equation (1) is Ulam-Hyers stable.

Theorem 4. Assume that (A1),(A3),(A4)and (4) hold. Suppose there exists an increasing function $\varphi \in C_{1-\gamma, \log }[J, X]$ and there exists $\lambda_{\varphi}>0$ such that for any $t \in J$

$$
I_{1+}^{\alpha} \varphi(t) \leq \lambda_{\varphi} \varphi(t) .
$$

Then, the problem (1) is Ulam-Hyers-Rassias stable.

Proof. Let $z \in C_{1-\gamma, \log }^{\gamma}[J, X]$ be solution of the inequality (8) and let $x \in C_{1-\gamma, \log }^{\gamma}[J, X]$ be the unique solution of the following problem

$$
\begin{aligned}
& { }_{H} D_{1^{+}}^{\alpha, \beta} x(t)=f(t, x(t), H x(t)), \quad t \in J:=[1, T], \\
& \left.I_{1^{+}}^{1-\gamma} x(t)\right|_{t=1}=\left.I_{1^{+}}^{1-\gamma} z(t)\right|_{t=1}=x_{0}, \quad \gamma=\alpha+\beta-\alpha \beta,
\end{aligned}
$$

where $0<\alpha<1$ and $0 \leq \beta \leq 1$.

We obtain the integral equation as follows:

$$
x(t)=x_{0}(t)+\left[I_{1^{+}}^{\alpha} f(s, x(s), H x(s))\right](t) .
$$

By integration of the inequality (8), we get

(10) $\left|z(t)-x_{0}(t)-\frac{1}{\Gamma(\alpha)} \int_{1}^{t}\left(\log \frac{t}{s}\right)^{\alpha-1} f(s, z(s), H z(s)) \frac{d s}{s}\right| \leq \epsilon \lambda_{\varphi} \varphi(t)$. 
On the other hand, we have

$$
\begin{aligned}
& \quad|z(t)-x(t)| \\
& \leq\left|z(t)-x_{0}(t)-\frac{1}{\Gamma(\alpha)} \int_{1}^{t}\left(\log \frac{t}{s}\right)^{\alpha-1} f(s, z(s), H z(s)) \frac{d s}{s}\right| \\
& \quad+\frac{L_{1}\left(1+H_{1}\right)}{\Gamma(\alpha)} \int_{1}^{t}\left(\log \frac{t}{s}\right)^{\alpha-1}|z(s)-x(s)| \frac{d s}{s} \\
& \leq \epsilon \lambda_{\varphi} \varphi(t)+\frac{L_{1}\left(1+H_{1}\right)}{\Gamma(\alpha)} \int_{1}^{t}\left(\log \frac{t}{s}\right)^{\alpha-1}|z(s)-x(s)| \frac{d s}{s} .
\end{aligned}
$$

By applying Lemma 2 and Remark 3, we get

$$
|z(t)-x(t)| \leq \epsilon \lambda_{\varphi} \varphi(t) E_{\alpha, 1}\left(L_{1}\left(1+H_{1}\right)(\log T)^{\alpha}\right), \quad t \in[1, T] .
$$

Thus, the equation (1) is generalized Ulam-Hyers-Rassias stable. The proof is completed.

\section{References}

[1] S. Abbas, M. Benchohra, S. Sivasundaram, Dynamics and Ulam stability for Hilfer type fractional differential equations, Nonlinear Stud., vol. 23, no. 4, 2016, 627-637.

[2] S. Abbas, M. Benchohra, N. Hamidi, Hilfer and HilferHadamard Fractional Differential Equations with Random Effects, Libertas Mathematica (new series), vol. 37, 2017, 45-64.

[3] S. Abbas, M. Banchohra, J. E. Lazreg, J. J. Nieto, On a coupled system of Hilfer- Hadamard fractional differential equations in Banach spaces, J. Nonlinear Funct. Anal. 2018, 2018, Article ID 12.

[4] B. Ahmad, S. K. Ntouyas, Initial value problems for hybrid Hadamard fractional differential equations, Electron. J. Differential Equations, vol. 161, 2014, 1-8.

[5] B. Ahmad, S. Sivasundaram, Some existence results for fractional integrodifferential equations with nonlocal conditions, Commun. Appl. Anal., vol. 12, 2008, 107-112.

[6] B. Ahmad, J. J. Nieto, Riemann-Liouville fractional differential equations with fractional boundary conditions, Fixed point Theory, vol. 13, 2013, 329-336.

[7] S. Andras, J. J. Kolumban, On the Ulam-Hyers stability of first order differential systems with nonlocal initial conditions, Nonlinear Anal. Theory Methods Appl., vol. 82, 2013, 1-11. 
[8] K. Balachandran, S. Kiruthika, J. J. Trujillo, Existence results for fractional impulsive integrodifferential equations in Banach spaces, Commun Nonlinear Sci. Numer. Simul., vol. 16, 2011, 1970-1977.

[9] K. Balachandran, S. Kiruthika, J. Y. Park, Controllability of fractional integrodifferential systems in Banach spaces, Nonlinear Anal. Hybri., vol. 3, 2009, 363-367.

[10] K. Balachandran, S. Kiruthika, Existence results for fractional integrodifferential equations with nonlocal condition via resolvent operators, Comput. Math. Appl., vol. 62, 2011, 1350-1358.

[11] P. L. Butzer, A. A. Kilbas, J. J. Trujillo, Compositions of Hadamard-type fractional integration operators and the semigroup property, J. Math. Anal. Appl., vol. 269, 2002, 1-27.

[12] P. L. Butzer, A. A. Kilbas, J. J. Trujillo, Mellin transform analysis and integration by parts for Hadamard-type fractional integrals, J. Math. Anal. Appl., vol. 270, 2002, 1-15.

[13] K. M. Furati, M. D. Kassim, N. E. Tatar, Existence and uniqueness for a problem involving Hilfer fractional derivative, Comput. Math. Appl., vol. 64, 2012, 1616-1626.

[14] K. M. Furati, M. D. Kassim, N. E. Tatar, Non-existence of global solutions for a differential equation involving Hilfer fractional derivative, Electron. J. Differential Equations, vol. 235, 2013, 1-10.

[15] H. Gu, J. J. Trujillo, Existence of mild solution for evolution equation with Hilfer fractional derivative, Appl. Math. Comput., vol. 257, 2014, 344-354.

[16] V. Gupta, J. Dabas, Existence of solution for fractional impulsive integrodifferential equation with integral boundary conditions, Func. Anal.-TMA, vol. 1, 2015, 56-68.

[17] V. Gupta, J. Dabas, Michal Feckan, Existence results of solutions for impulsive fractional differential equations, Nonauton. Dyn. Syst., vol. 5, 2018, 35-51.

[18] R. W. Ibrahim, Generalized Ulam-Hyers stability for fractional differential equations, Int. J. Math., vol. 23, 2012, 1250056.

[19] S. M. Jung, Hyers-Ulam stability of linear differential equations of first order, Appl. Math. Lett., vol. 17, 2004, 1135-1140.

[20] R. Kamocki, C. Obcznnski, On fractional Cauchy-type problems containing Hilfer derivative, Electron J. Qual. Theory Differ. Equ., vol. 50, 2016, 1-12. 
[21] K. Karthikeyan, B. Ahmad, Existence results for boundary value problems of arbitrary order integrodifferential equations in Banach spaces, An. St. Univ. Ovidius Constant a, vol. 21, no. 2, 2013, 155-171, DOI: 10.2478/auom-20130029 .

[22] K. Karthikeyan, J. J. Trujillo, Existence and uniqueness results for fractional integrodifferential equations with boundary value conditions, Commun. Nonlinear Sci. Numer. Simul., vol. 17, 2012, 4037-4043.

[23] M. D. Kassim, N. E. Tatar, Well-psedness and stability for a differential problem with Hilfer-Hadamard fractional derivative, Abstr. Appl. Anal., vol. 1, 2013, 112 .

[24] P. Muniyappan, S. Rajan, Hyers-Ulam-Rassias stability of fractional differential equation, Int. J. Pure Appl. Math., vol. 102, 2015, 631-642.

[25] Y. Rian, S. Shurong, S. Ying, H. Zhenlai, Boundary value problems for fractional differential equations with nonlocal boundary conditions, Adv. Differ. Equ., vol. $176,2013$.

[26] I. A. Rus, Ulam stabilities of ordinary differential equations in a Banach space, Carpathian J. Math., vol. 26, 2010, 103-107.

[27] R. Hilfer, Application of fractional Calculus in Physics, World Scientific, Singapore, 1999.

[28] R. Hilfer, Y. Luchko, Z. Tomovski, Operational method for the solution of fractional differential equations with generalized Riemann-Lioville fractional derivative, Fract. Calc. Appl. Anal., vol. 12, 2009, 289-318.

[29] J. R. Wang, Y. Zhang, Nonlocal initial value problems for differential equations with Hilfer fractional derivative, Appl. Math. Comput., vol. 266, 2015, 850-859.

[30] A. A. Kilbas, H. M. Srivastava, J. J. Trujillo, Theory and Applications of Fractional Differential Equations, in: Mathematics Studies, Elsevier, vol. 204, 2006.

[31] S. G. Samko, A. A. Kilbas, O. I. Marichev, Fractional Integrals and Derivatives, Theory and Applications, Gordon and Breach, Amsterdam, Engl. Trans.from the Russian, 1987.

[32] I. Podlubny, Fractional Differential Equations, in: Mathematics in Science and Engineering, Acad. Press, vol. 198, 1999.

[33] D. Vivek, K. Kanagarajan, S. Sivasundaram, Dynamics and stability of pantograph equations via Hilfer fractional derivative, Nonlinear Stud., vol. 23, no. 4, 2016, 685-698. 
[34] J. Wang, L. Lv, Y. Zhou, Ulam stability and data dependence for fractional differential equations with Caputo derivative, Electron J. Qual. Theory Differ. Equ., vol. 63, 2011, 1-10.

[35] J. Wang, Y. Zhou, New concepts and results in stability of fractional differential equations, Commun. Nonlinear Sci. Numer. Simulat., vol. 17, 2012, 2530-2538.

[36] J. Wang, Y. Zhou, M. Medved, Existence and stability of fractional differential equations with Hadamard derivative, Topol. Methods Nonlinear Anal., vol. 41, no. 1, 2013, 113-133.

\section{Vivek}

Department of Mathematics

Sri Ramakrishna Mission Vidyalaya College of Arts and Science

Coimbatore-641020, India.

e-mail: peppyvivek@gmail.com

\section{K. Kanagarajan}

Department of Mathematics

Sri Ramakrishna Mission Vidyalaya College of Arts and Science

Coimbatore-641020, India.

e-mail: kanagarajank@gmail.com

\section{E. M. Elsayed}

Department of Mathematics

Faculty of Science

King Abdulaziz University

Jeddah 21589, Saudi Arabia.

e-mail: emmelsayed@yahoo.com 